\title{
STABILIZATION OF BALLOONING MODES WITH SHEARED TOROIDAL ROTATION
}

\author{
by \\ R.L. MILLER, F.W. WAELBROECK, L.L. LAO, \\ and T.S. TAYLOR
}

NOVEMBER 1994 
This report was prepared as an account of work sponsored by an agency of the United States Government. Neither the United States Government nor any agency thereof, nor any of their employees, makes any warranty, express or implied, or assumes any legal liability or responsibility for the accuracy, completeness, or usefulness of any information, apparatus, produce, or process disclosed, or represents that its use would not infringe privately owned rights. Reference herein to any specific commercial product, process, or service by trade name, trademark, manufacturer, or otherwise, does not necessarily constitute or imply its endorsement, recommendation, or favoring by the United States Government or any agency thereof. The views and opinions of authors expressed herein do not necessarily state or reflect those of the United States Government or any agency thereof. 


\section{DISCLAIMER}

Portions of this document may be illegible in electronic image products. Images are produced from the best available original document. 


\title{
STABILIZATION OF BALLOONING MODES WITH SHEARED TOROIDAL ROTATION
}

\author{
by \\ R.L. MILLER, F.W. WAELBROECK, ${ }^{\star}$ L.L. LAO, \\ and T.S. TAYLOR
}

This is a preprint of a paper to be presented at the Fifteenth International Conference on Plasma Physics and Controlled Nuclear Fusion Research, September 26-October 1, 1994, Seville, Spain, and to be printed in the Proceedings.

\author{
Work supported by \\ U.S. Department of Energy \\ Grant No. DE-FG03-92ER54150
}

*Institute for Fusion Studies

GENERAL ATOMICS PROJECT 3938 NOVEMBER 1994

DISTRIBUTION OF THIS DOOUMENT IS LNLIMTED ${ }^{w}{ }^{w}$ 
IAEA-CN-60/D-1-1-3-1

\section{STABILIZATION OF BALLOONING MODES WITH SHEARED TOROIDAL ROTATION}

\section{ABSTRACT}

A new code demonstrates the stabilization of MHD ballooning modes by sheared toroidal rotation. A shifted-circle model is used to elucidate the physics and numerically reconstructed equilibria are used to analyze DIII-D discharges. In the ballooning representation, the modes shift periodically along the field line to the next point of unfavorable curvature. The shift frequency $(\mathrm{d} \Omega / \mathrm{d} q$ where $\Omega$ is the angular toroidal velocity and $q$ is the safety factor) is proportional to the rotation shear and inversely proportional to the magnetic shear. Stability improves with increasing shift frequency and, in the shifted circle model, direct stable access to the second stability regime occurs when this frequency is a fraction of the Alfvén frequency $\omega_{A}=V_{A} / q R$. Shear stabilization is also demonstrated for an equilibrium reconstruction of a DIII-D VH-mode.

\section{INTRODUCTION}

The maximum achievable value of beta (the ratio of thermal to magnetic energy) is determined by Magneto-hydrodynamic (MHD) stability. In unbalanced neutral-beam heated discharges $[1,2]$, however, the rotation energy can be a significant fraction of the thermal energy of the plasmas and can influence MHD behavior.

We investigate the evolution of short wavelength perturbations with broad extent across flux surfaces. Such perturbations are very general; they may describe non-stationary (convective) amplification as well as arrays of locally resonant eigenmodes [3]. Convective amplification is of particular concern in cases where the growth rate estimated by neglecting the effect of rotation is larger than the rotation shear frequency.

We consider equilibria with purely toroidal rotation,

$$
\bar{v}=R \Omega(\psi) \hat{\varphi}
$$

where $R$ is the major radius, $\hat{\varphi}$ is a unit vector in the toroidal direction and the rotation frequency $\Omega(\psi)$ is a function of the poloidal flux $\psi$. The perturbations are described by [4]

$$
\tilde{A} \rightarrow \tilde{A} e^{i n(\varphi-q \theta-\Omega t)}
$$

where $\varphi$ is the toroidal angle, $\theta$ is a poloidal angle defined to make field lines "straight" and $q$ is the safety factor. Unlike for static ballooning modes, the eikonal is time dependent; we must solve an initial value problem. 
Loss of confinement results most directly from convection between the core and the plasma boundary. This occurs when the phase of the eikonal is constant across the flux surfaces, or for $\theta=(d \Omega / d q) t$. As a result the extended eddy experiences alternately the effect of favorable and unfavorable curvature. This is the primary cause of stabilization by sheared rotation for toroidal instabilities.

In Section II we present the approximations used in the shifted circle model. In Section III numerical solutions to shifted circle equations provide an $s-\alpha$ diagram. Section IV presents numerical solutions for DIII-D equilibria and Section V is a summary of results and conclusions.

\section{EQUATIONS}

The linear perturbation of an equilbrium with sheared toroidal rotation is described by the stream function $\chi$, the poloidal flux $\psi$, the velocity along the field line $v_{\| l}$, the pressure $\mathrm{p}$ and the mass density $p$. The stream function $\chi$ is related to the electrostatic and magnetic potentials by $\chi=(\phi+\bar{A} \cdot \bar{v}) / B$. We assume an isothermal equation of state.

The exact ballooning mode equations which are solved in Section IV are given in Miller et al. [5]. Here, the standard shifted-circle model is obtained by considering a low-beta equilibrium in which the pressure varies rapidly in a thin radial shell [6]. We generalize this approach by assuming that the velocity is small but changes rapidly in the same region. The density of kinetic energy is typically no greater than $20 \%$ of the pressure in present experiments. The shear of the velocity, by contrast, is comparable to the magnetic shear in the core. Accordingly, we neglect centrifugal forces for the shifted circle equilibrium and focus instead on the velocity shear. equations

In dimensionless form, our model equilibrium results in the ballooning mode

$$
\begin{gathered}
\partial_{\tau} \hat{\mathrm{v}}_{\|}=-\sigma(\theta) \hat{\chi}+\partial_{\theta} \hat{p}-L_{p_{0}}^{-1} \hat{\psi} \\
\partial_{\tau}\left[\hat{\chi}\left(1+h^{2}(\theta, \tau)\right)\right]=2 q^{2} \Gamma(\theta, \tau) \hat{p}-V_{A}^{2} \partial_{\theta}\left[\hat{\psi}\left(1+h^{2}(\theta, \tau)\right)\right] \\
\partial_{\tau} \hat{\psi}=-\partial_{\theta} \hat{\chi} \\
\partial_{\tau} \hat{p}=-L_{p_{0}}^{-1} \hat{\chi}+\left[\partial_{\theta} \hat{\mathrm{v}}_{\|}-2 \Gamma(\theta, \tau) \hat{\chi}-\sigma(\theta) \hat{\psi}\right]
\end{gathered}
$$

where $h(\theta, \tau)=s \theta-s_{v} \tau-\alpha \sin \theta$ is proportional to the integral of the local magnetic shear along the field-line, $1+h^{2}$ is proportional to the norm of the wavevector, $\Gamma(\theta, \tau)=\cos \theta+\sin \theta h(\theta, \tau)$ is proportional to the triple product of the curvature, wavevector and magnetic field, and $\sigma(\theta)=s_{v}\left(\varepsilon^{-1}+2 \cos \theta\right)$ determines the convective acceleration resulting from field-line interchange. We have 
normalized time to the sound wave transit frequency and distances to the connection length, $\tau=t / t_{0}=t V_{T} / q R_{0}$, where $V_{T}=\sqrt{T / m}$. As a result the Alfvén velocity parameter $\mathrm{V}_{\mathrm{A}}$ is inversely proportional to the square root of beta. The perturbation amplitudes have been normalized according to $\hat{\mathrm{v}}_{\|}=\mathrm{v}_{\|} B / \rho_{0} V_{T}, \hat{\chi}=\chi q^{2} / r B V_{T}$, $\hat{\psi}=\tilde{\psi} q^{2} / r B^{2}, \hat{p}=\tilde{p} / p_{0}(\psi)$. Lastly, $L_{p_{0}}^{-1}=R_{0} \partial_{\psi} \ln p_{0}(\psi) \partial_{r} \psi$.

The three parameters of interest are the pressure gradient $\alpha=-2 q^{2} / L_{p_{0}} V_{A}^{2}$, the magnetic shear $s=r \partial_{r} q / q$, and the velocity shear $s_{v}=R_{0} r \partial_{r} \Omega / V_{T}$. Note that values of $s_{\mathrm{V}}$ of order 1 have been observed in VH-mode plasmas in DIII-D [1]. With sheared rotation, as can be seen from Eqs. (3-6), the $s-\alpha-s_{V}$ diagram depends on three subsidiary parameters: $\mathrm{V}_{\mathrm{A}}, q$ the safety factor, and $\varepsilon$ the inverse aspect ratio. In the next Section we will take $q=2, \varepsilon=0.1$ and set $V_{A}=\sqrt{40}$, corresponding to a $\beta$ value of $5 \%$

As pointed out by Hameiri and Chun, $[\mathrm{H} \& \mathrm{C}]$ the equations are periodic in time in a coordinate system precessing along the field-line, $\eta=\theta-\left(s_{v} / s\right) \tau$. It follows from the Floquet theorem that the solution has the form [7]

$$
X(\eta, \tau)=\sum_{i} x_{i}(\eta, \tau) e^{\gamma_{i} \tau}
$$

where the Floquet characteristic functions $x_{i}(\eta, \tau)$ are periodic, $x_{i}(\eta, \tau)=x_{i}(\eta, \tau+T)$, and the growth rates $\gamma_{i}$ of the individual Floquet modes may be complex. There is a direct correspondence between the Floquet characteristic functions and the radial eigenfunctions, and the Floquet growth rates are equal to the eigenvalues of the normal modes [3]. quantity

In most of the present work the growth rate is obtained from the integrated

$$
\Lambda(t)=\sqrt{\int_{\theta_{\min }}^{\theta_{\max }}\left(\hat{X}^{2}+\hat{Z}^{2}+\hat{\psi}^{2}+\hat{p}^{2}\right) d \theta}
$$

by calculating the logarithm of the amplification during a period $T$, where $T=2 \pi s / s_{v}$. That is,

$$
\gamma=(1 / T) \ln [\Lambda(t+T) / \Lambda(t)]
$$

A more rigorous approach using Floquet's theorem has also been used. One can identify the dominant $\gamma_{i}$ eigenvalue and its complex conjugate from the $3 \times 3$ determinant

$$
\left|\begin{array}{ccc}
1 & x\left(\eta_{1}, \tau\right) & x\left(\eta_{2}, \tau\right) \\
e^{\gamma \tau} & x\left(\eta_{1}, \tau+T\right) & x\left(\eta_{2}, \tau+T\right) \\
e^{2 \gamma T} & x\left(\eta_{1}, \tau+2 T\right) & x\left(\eta_{2}, \tau+2 T\right)
\end{array}\right|=0
$$




\section{SOLUTION OF THE EQUATIONS}

To solve the set of equations (3-6) we have written an initial value code which uses two-step Lax-Wendroff differencing [8]. Initial conditions are obtained by starting without rotation shear and letting an unstable ballooning mode form.

\section{A. MODE STRUCTURE}

The evolution of the perturbed pressure as a function of theta is shown in Fig. 1 at six different times for $s=0.5, \alpha=1$, and $s_{\gamma}=0.25$. The mode initially remains in the bad-curvature region, despite the increase of the phase shift between neighboring poloidal harmonics. As the ballooning-angle increases further, the mode eventually shifts along the field line to the next point of unfavorable curvature where its poloidal harmonics may again be in phase (Fig. 1). The process is then repeated.
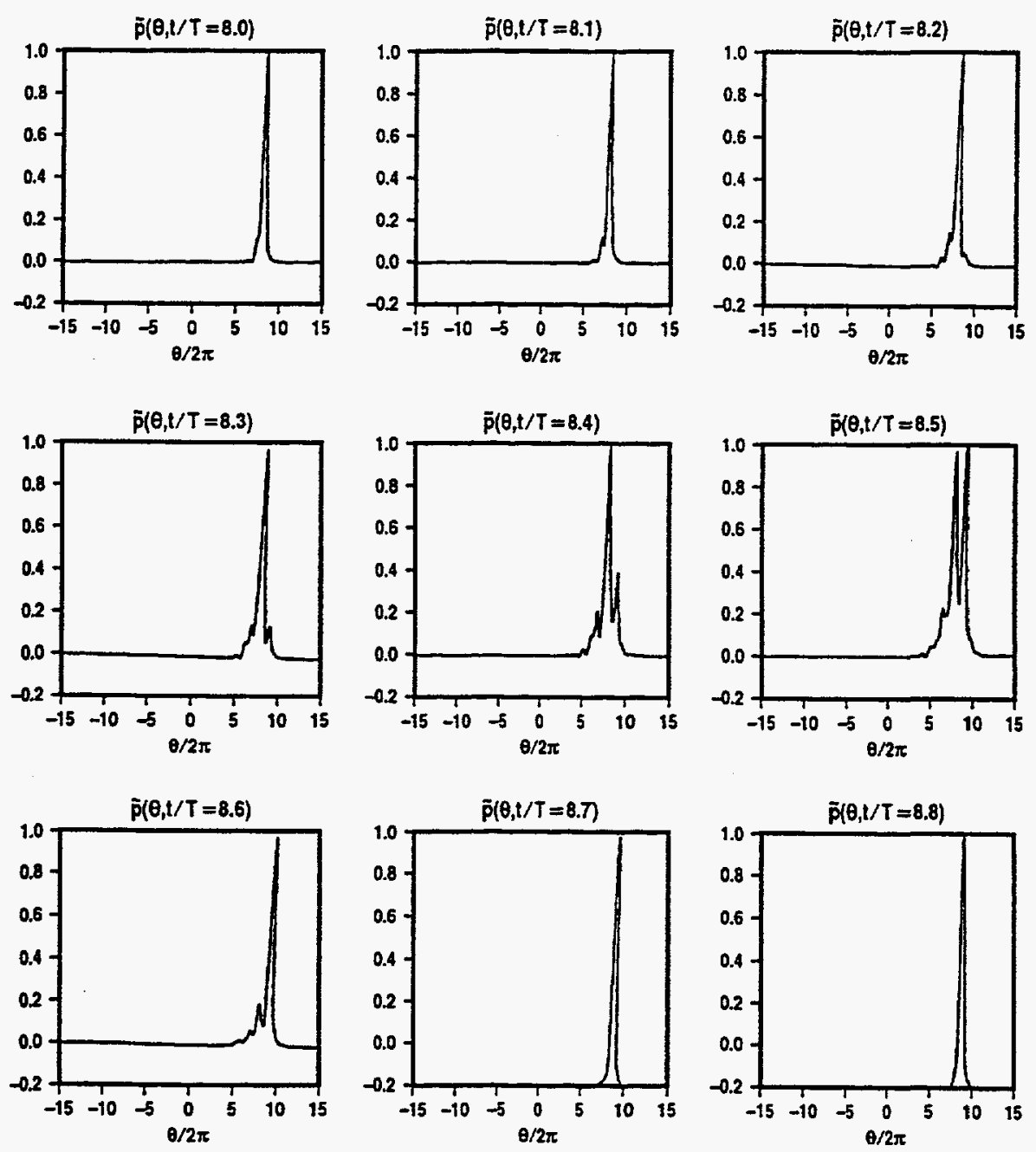

Fig. 1. Evolution of the pressure profile $p(\theta, t)$ for $s=0.5, \alpha=1, s_{v}=0.25$. 
In Fig. 2 we show the growth rate as a function of $\left(\mathrm{s}_{\mathrm{v}} / \mathrm{s}\right) / \mathrm{V} \mathrm{A}$ (the dimensionless form of $d \Omega / d q$ ) with $s=1$ and $\alpha=2$ for $\beta=1.6 \%$ and $5 \%$. The additional curve corresponds to an incompressible model obtained by neglecting the bracketed term on the right hand side of Eq. (6).

Three conclusions can be drawn from Fig. 2. First, the structure of the $\beta=1.6 \%$ and $5 \%$ curves is clearly due to compressibility and the sound wave. Second, stabilization occurs at a lower value of $s_{\mathrm{V}}$ in the compressible model. Third, increasing beta is stabilizing at least up to $\beta=5 \%$.

For magnetic shear $s<1$, motion of the mode center is not as important, because the mode is not as well localized in $\theta$; weak shear is more easily stabilized than strong shear for a given $\mathrm{s}_{\mathrm{v}}$.

\section{B. $s-\alpha$ DIAGRAM}

We have constructed a modified s-alpha diagram for $s_{\mathrm{V}}=1$ and $\mathrm{s}_{\mathrm{V}}=2$. The data was obtained by choosing 11 values of magnetic shear, $s=\{0.1,0.2,0.4,0.6,0.8,1.0$, $1.2,1.4,1.6,1.8,2.0\}$, and scanning alpha in increments of 0.1 over the entire range for which there exists an unstable static mode. The results are shown in Fig. 3 We see that the unstable region is eroded on both the first and second stable sides. For small enough magnetic shear $s$, complete elimination of the unstable region results. In this range of $\beta(\sim 5 \%)$, a rough criterion for complete stabilization is

$$
\frac{s_{\mathrm{v}}}{\mathrm{s}} \geq(1 / 3) \mathrm{V}_{\mathrm{A}}
$$

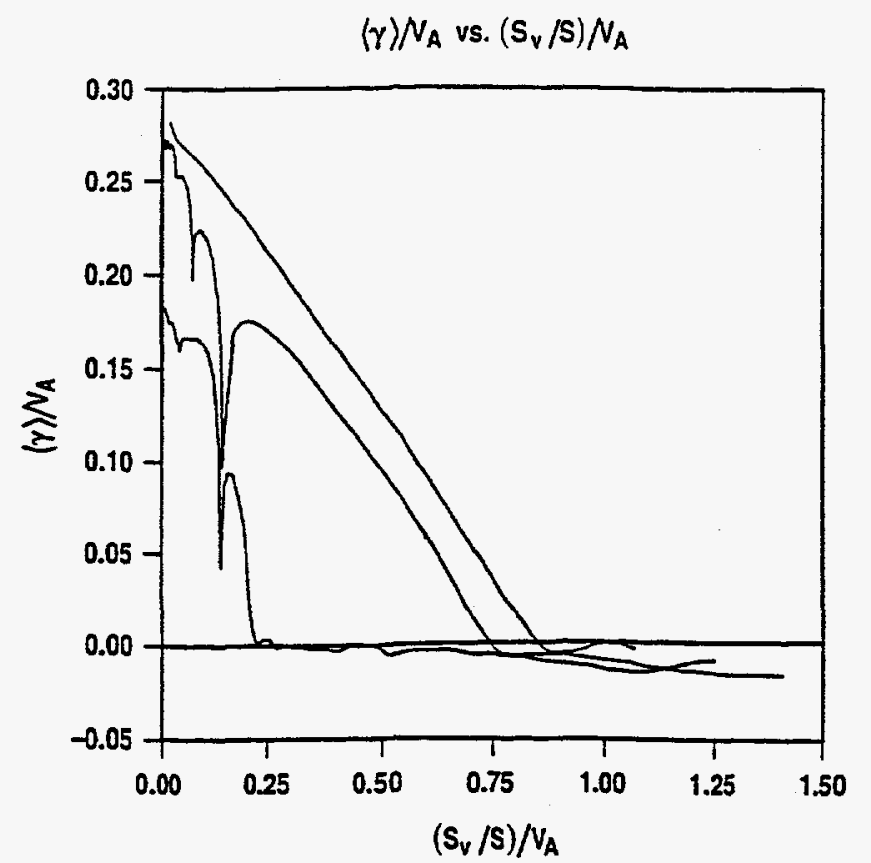

Fig. 2. Growth rate $\gamma / V_{A}$ versus $\left(s_{\gamma} / s\right) / V_{A}$ with $s=1$ and $\alpha=2$ for $\beta=\{0.5 \%, 1.6 \%, 5 \%, 16 \%\}$ and the incompressible model. 


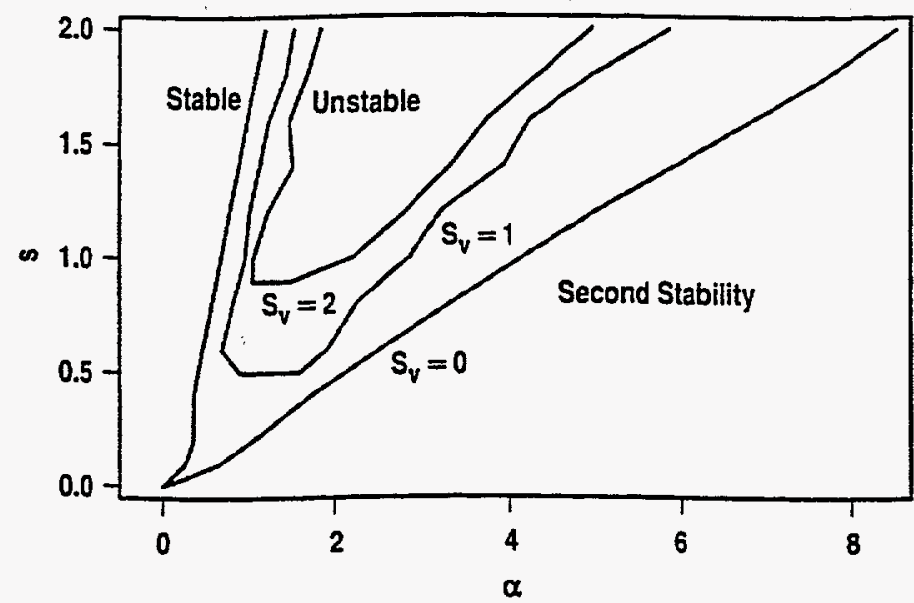

Fig. 3. $s-\alpha$ diagram for two different values of sheared toroidal rotation: $s_{\nu}=1$ and $s_{v}=2$.

or

$$
\frac{\mathrm{d} \Omega}{\mathrm{d} q} \geq(1 / 3) \frac{\bar{V}_{A}}{\mathrm{R}_{\mathrm{o}} q}
$$

where $\bar{V}_{A}$ is the actual Alfvén velocity and we have used $s_{v} / s=R_{o} q[(d \Omega / d q) /$ $\left.V_{T}\right]$. For weak values of magnetic shear, $s \leq 0.1$ the coefficient $1 / 3$ in Eq. (11) should be replaced by a value approaching one.

\section{NUMERICAL EQUILIBRIA AND STABILITY}

The equilibrium reconstruction code EFIT has been modified to include toroidal rotation. The code uses data from a 16 channel motional-Stark effect diagnostic and external magnetic data. The pressure and toroidal rotation profiles are constrained by the measured kinetic profile and the charge exchange recombination diagnostic, respectively. The most distinctive feature of these equilibria is the diplacement of the pressure surfaces from the magnetic flux surfaces.

Using EFIT and the initial value ballooning code, we have examined the ballooning stability of a DIII-D VH-mode discharge [1] in some detail. The rotation energy is $10 \%$ of the thermal energy. EFIT was used to construct equilibria both with and without plasma rotation. The ballooning stability was then determined for the two equilibria using the initial value code. The ballooning results of the static case as well as other static cases have been checked with the existing ballooning mode codes, $\mathrm{MBC}$ and CAMINO, and found to be in reasonable agreement.

All flux surfaces were stable for the equilibrium reconstruction of the discharge without rotation, while 2 out of 41 flux surfaces were found to be unstable for the equilibrium reconstruction with rotation. These unstable flux surfaces were near the center of the plasma; the percentage of the total poloidal flux on these surfaces was 13-15\%. Unlike the shifted circle model, this analysis includes the effects of finite toroidal rotation (centrifugal and Coriolis effects). The instability appears to arise from an interaction between the rotation and the rotation shear. 
The dimensionless parameters for the two unstable flux surfaces were $(\mathrm{s}=$ $\left.0.063, \mathrm{~s}_{\mathrm{v}}=0.164, \mathrm{~V}_{\mathrm{A}}=5.60, \mathrm{~s}_{\mathrm{v}} /\left(\mathrm{s} \mathrm{V}_{\mathrm{A}}\right)=0.46\right)$ and $\left(\mathrm{s}=0.108, \mathrm{~s}_{\mathrm{v}}=0.207, \mathrm{~V}_{\mathrm{A}}=5.74\right.$, $\left.s_{\mathrm{v}} /\left(s \mathrm{~V}_{\mathrm{A}}\right)=0.33\right)$. The criterion of Eq. (11) suggests that both of these flux surfaces should have been shear stabilized. However, Eq. (11) is not expected to hold for such low values of magnetic shear. We find that the $s=0.063$ flux surface can be stabilized by a $25 \%$ increaes in $\mathrm{s}_{\mathrm{v}}$ or by a $25 \%$ decrease in $\mathrm{s}$, which gives a value of $\mathrm{s}_{\mathrm{v}} /\left(\mathrm{s} \mathrm{V}_{\mathrm{A}}\right)=$ 0.58 . The $\mathrm{s}=0.108$ surface was more difficult to stabilize, requiring an increase of $\mathrm{sv}$ by $60 \%$ or a decrease of $s$ by $50 \%$, with a resulting $\mathrm{s}_{\mathrm{v}} /\left(\mathrm{s} \mathrm{V}_{\mathrm{A}}\right)$ of 0.53 to 0.66 . Note that in such a weak shear region, an adjustment of the magnetic shear by these amounts has a negligible effect on the $q$ profile, suggesting that stable profiles can be found within the experimental range of error.

Although the profile changes required here for stabilization were modest, this case demonstrates that rotation can influence ballooning stability.

\section{SUMMARY \& CONCLUSIONS}

The initial value ballooning code demonstrates stabilization of MHD ballooning modes by sheared toroidal rotation. We have developed a shifted-circle equilibrium model and extended the $s-\alpha$ diagram to describe the effect of sheared toroidal rotation. We find that the unstable region of the s- $\alpha$ diagram is reduced on both the first- and second-stable sides by sheared toroidal rotation. The dominant parameter determining where the unstable region is eliminated is $\left(s_{v} / s\right) / V_{A}$ or $(d \Omega / d q) R_{0} q / \bar{V}_{A}$ where $\bar{V}_{A}$ is the actual Alfvén velocity.

Using the full set of equilibrium and ballooning mode equations including toroidal rotation and toroidal rotation shear we have examined the stability of numerically reconstructed equilibria. Although some destabilization can occur through an interaction between the rotation and rotation shear, once again we find that increasing the rotation shear is stabilizing.

\section{ACKNOWLEDGEMENT}

This work was supported by the U.S. Department of Energy under Grant No. DE-FG03-92ER54150.

\section{REFERENCES}

[1] GREENFIELD, C.M., et al., Plasma Phys. and Contr. Fusion 35, (1993) B263.

[2] SCOTT, S.D., et al., Phys. Rev. Lett. 64, (1990) 531.

[3] WAELBROECK, F. and CHEN, L., Phys. Fluids B 3 (1991) 601.

[4] COOPER, W.A., Plasma Physi. and Contr. Fusion 30 (1988) 1805.

[5] MILLER, R.L., WAELBROECK, F.L., HASSAM, A.B., and WALTZ, R.E., General Atomics Report GA-A21751, submitted to Physics of Plasmas.

[6] CONNOR, J.W., HASTIE, R.J., TAYLOR, J.B., Phys. Rev. Lett. 40 (1978) 396.

[7] HAMEIRI, E., and CHUN, S.T., Phys. Rev. A 41 (1990) 1186.

[8] PRESS, W.H., TEUKOLSKY, S.A., VETTERLING, W.T., and FLANNERY, B.P., Numerical Recipes in Fortran, Second Edition, Cambridge University Press, 1992.

[9] LAZARUS, E.A., et al., Phys. Fluids B 4, (1992) 3644. 